\title{
Polarisable Hydrogen Bond Formation and Ionic Interactions in Model Phospholipid Polar Head Molecules
}

\author{
Georg Papakostidis and Georg Zundel
}

Physikalisch-Chemisches Institut der Universität München

(Z. Naturforsch. 28 b, 323-330 [1973]; received March 5, 1973)

Ionic interactions, phospholipids, polarisability, hydrogen bonds, IR spectra.

The serine phosphoric acid P-methylester (SPM) and the ethanol-amine phosphoric acid P-methylester (EPM) were synthesized as water soluble models for the functional groups of the corresponding phospholipids. Investigations were made of the aqueous solutions of these molecules as a function of deprotonation and protonation. An intramolecular, easily polarisable hydrogen bond occurs in the zwitterion of the SPM. The solutions of different salts of SPM were studied as well as the influence of counterion pairs. Counterion pairs hardly influence these bonds. At about 50\% deprotonation extremely easily polarisable intermolecular bonds form. At about $100 \%$ deprotonation of the zwitterion the hydrogen bonds observed are affected by the presence of $\mathrm{CO}_{2}$. The above is indicated by changes of the bands of the carboxylic and phosphate groups, and in particular by a continuous absorption in the infrared spectrum. During protonation of the EPM easily polarisable intermolecular $\mathrm{POH}^{+} \ldots$. OP hydrogen bonds form at first, but as protonation increases the solutions become acidic, that is, $\mathrm{H}_{5} \mathrm{O}_{2}{ }^{+}$groupings form.

\section{Introduction}

Numerous papers deal with interactions of ions with phospholipids ${ }^{1-6}$. The difficulty with all these investigations was due to the fact that either micella were present or that the phospholipids had to be studied in a non-aqueous medium. The functional groups of the phospholipids phosphatidyl serine and phosphatidyl etanolamine are probably present in the hydrophilic regions of the membranes. However, in these regions the immediate environment of these groups hardly differs from that in aqueous medium. Therefore water soluble model molecules were synthesized for the functional groups of these phospholipids? ${ }^{7}$ namely the serine phosphoric acid P-methylester (SPM) and the etanolamine phosphoric acid P-methylester (EPM).<smiles>COP(=O)(O)OCC([NH3+])C(=O)O</smiles>

Requests for reprints should be sent to Dr. G. ZUNDEL, Physikal.-Chem. Institut d. Univ., D-8000 München 2, Theresienstr. 41.<smiles>C[PH]([O-])([O-])OCC[NH3+]</smiles>

The IR spectra of aqueous solutions of these molecules do not change greatly on varying the cations present $\left(\mathrm{Na}^{+}, \mathrm{K}^{+}, \mathrm{Ca}^{2+}\right.$ or $\left.\mathrm{Mg}^{2+}\right)$. However, it is evident that these molecules form extremely easily polarisable hydrogen bonds.

Hydrogen bonds with a double minimum potential well or with a flat, very broad potential are extremely easily polarisable $\mathbf{8 , 9}$. This polarisability gives rise to numerous kinds of interaction effects of these hydrogen bonds with their environment, for instance, an induced dipole interaction of these hydrogen bonds with the fields of the ions and the dipole fields of the solvate molecules. All these interactions cause a continuous energy level distribution of the protons in these hydrogen bonds, which is indicated by a continuous absorption in the IR spectrum (for further details see $\left.{ }^{8-15}\right)$. In the case of symmetrical hydrogen bonds $\mathrm{BH}^{+\cdots} \mathrm{B}$ these continua extend over the entire range from approx. $3000 \mathrm{~cm}^{-1}$ toward small wave number values ${ }^{9,10}$. In contrast, asymmetrical hydrogen bonds with a double minimum potential well cause continua in the range 
$3000-1700 \mathrm{~cm}^{-1}$, and sometimes in the range below $1000 \mathrm{~cm}^{-1} 9,14$.

\section{Results and Discussion}

SPM and EPM occur in aqueous solutions as zwitterions. The following series of measurements were carried out: Firstly, with the SPM the protons at the negatively charged fixed ions were replaced by adding $\mathrm{NaOH}$ solutions of increasing concentration, i.e. increasing degree of deprotonation (Fig. 1). Secondly, the zwitterion of the SPM and EPM was protonated by adding $\mathrm{HCl}$. Thirdly, the $\mathrm{Na}^{+}, \mathrm{K}^{+}$, $\mathrm{Ca}^{2+}$ and $\mathrm{Mg}^{2+}$ salts of the SPM were studied. Finally, EPM and the various salts of SPM were investigated
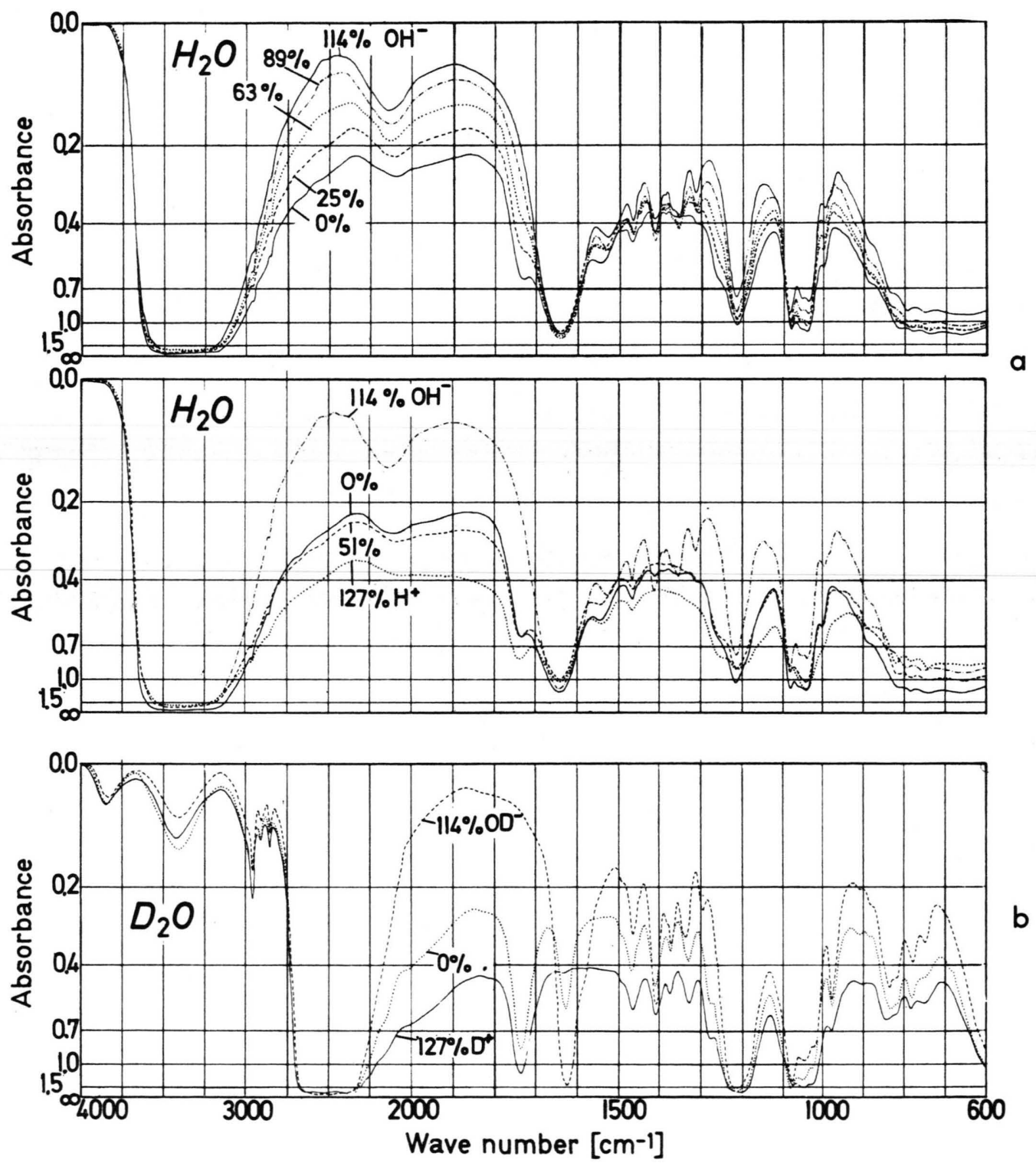

Fig. 1. IR Spectra of the aqueous serine phosphoric acid $\mathrm{P}$-methylester solutions as a function of $\%$ protonation and \% deprotonation with $\mathrm{HCl}$ or $\mathrm{NaOH}$ relative to the dissolved substance; a. $\mathrm{H}_{2} \mathrm{O}$ solutions; b. $\mathrm{D}_{2} \mathrm{O}$ solutions. 
in the stoichiometric presence of counterion pairs $\mathrm{NaCl}, \mathrm{KCl}, \mathrm{CaCl}_{2}$ and $\mathrm{MgCl}_{2}$ (for preparation see section III).

\section{II.1. Serine phosphoric acid P-methylester}

Fig. 1a shows the spectra of aqueous SPM solutions as a function of deprotonation and protonation. Fig. $1 \mathrm{~b}$ illustrates the spectra of corresponding $\mathrm{D}_{2} \mathrm{O}$ solutions. On deprotonation or protonation strong continua dissappear or appear, respectively.

With the zwitterion the continuum extends from approx. $3000-1800 \mathrm{~cm}^{-1}$ and then occurs again in the range below $1000 \mathrm{~cm}^{-1}$. Such a continuum is characteristic of an asymmetrical, easily polarisable hydrogen bond with a double minimum potential well. This type of hydrogen bond may form between the carboxylic and the phosphate groups, as illustrated by the calotte model shown in Fig. 2 .

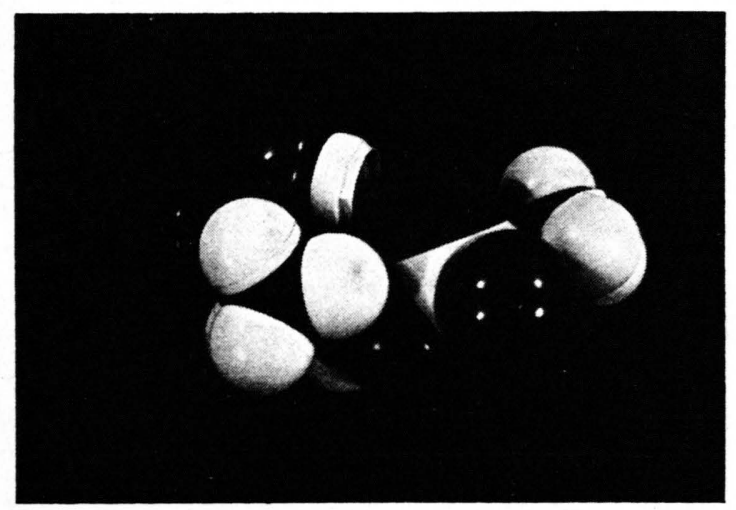

Fig. 2. Model of the serine phosphoric acid P-methylester molecule.

The bands of the anionic groups are summarised in Table I. As the proton approaches these groups, PO

Tab. I. The bands of the anionic groups.

$\left.\begin{array}{ll}1732 \mathrm{~cm}^{-1} & v \mathrm{C}=\mathrm{O} \\ 1628 \mathrm{~cm}^{-1} & v_{\mathrm{as}} \\ 1414 \mathrm{~cm}^{-1} & v_{\mathrm{s}}\end{array}\right\}$ in $-\mathrm{C} / \mathrm{OH}$

or $\mathrm{CO}$ bonds with double or single bond character form instead of two bonds of approximately equal strength. Instead of $\nu_{\mathrm{as}}$ and $\nu_{\mathrm{s}}$ of the $>\mathrm{PO}_{2}$ and $-\mathrm{CO}_{2}$ groups the stretching vibration of the double bond is then observed. Conversely, these bands indicate at which group the proton is located. In the spectrum of the zwitterion intense bands of $\boldsymbol{v}_{\mathrm{as}}$ and $\nu_{\mathrm{B}}$ of the $>\mathrm{PO}_{2}$ group are found at 1218 and 1084 $\mathrm{cm}^{-1}$, and a weak shoulder of $\nu \mathrm{P}=\mathrm{O}$ at $1252 \mathrm{~cm}^{-1}$. In the spectrum of the $\mathrm{D}_{2} \mathrm{O}$ solution an intense band of $\nu \mathrm{C}=\mathrm{O}$ is observed at $1732 \mathrm{~cm}^{-1}$ and a less intense band of $\nu_{\mathrm{as}}$ of the $-\mathrm{CO}_{2}$ group at $1628 \mathrm{~cm}^{-1}$. All this indicates that the proton can be present at both anions. This again strongly suggests that the continuum is caused by the postulated intramolecular hydrogen bond with a double minimum potential well. Moreover, the relative intensities of the bands show that the deeper well of the double minimum potential is located at the carboxylic group. This hydrogen bond is thus represented by both proton boundary structures $\mathrm{COH}^{+\cdots} \mathrm{OP}$ and $\mathrm{CO} \cdots \mathrm{H}^{+} \mathrm{OP}$, the former being of greater weight.

In view of these results the $\mathrm{pK}_{\mathrm{a}}$ values of the carboxylic and the phosphate groups can not differ strongly from each other. Fig. 3 shows a titration

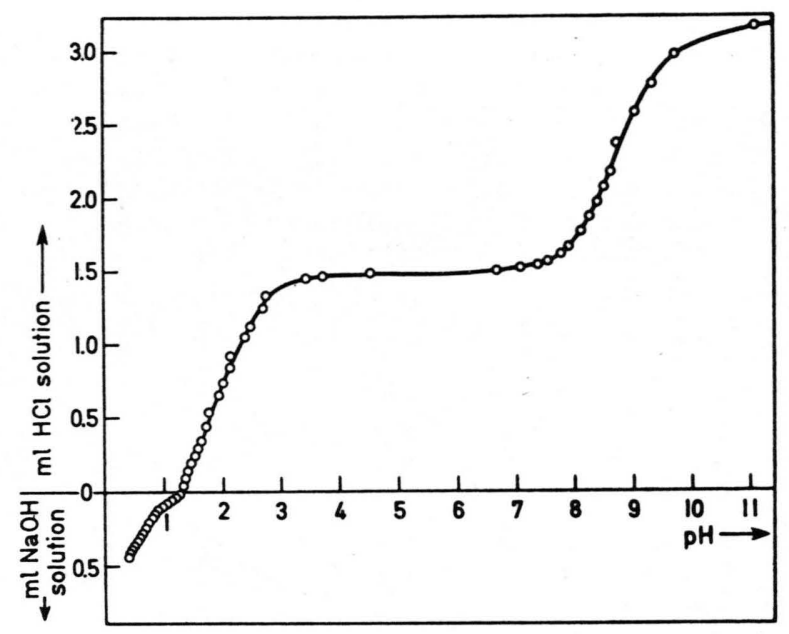

Fig. 3. Titration curve of an $1 \mathrm{~N}$ aqueous phosphoric acid P-methylester solution.

curve of an $1 \mathrm{~N}$ aqueous SPM solution. The rise in the $\mathrm{pH}$ range $8-10$ is caused by the deprotonation of the $-\mathrm{NH}_{3}$ group. The $\mathrm{pK}_{\mathrm{a}}$ of this group is much larger than the $\mathrm{pK}_{\mathrm{a}}$ of the carboxylic and of the phosphate groups, as indicated by the $\mathrm{pK}_{\mathrm{a}}$ valuse of the phospholipids given in l.c. ${ }^{6}$. This is shown by the extended plateau in the $\mathrm{pH}$ range $8-3$. The increase of the $\mathrm{pH}$ in the range $1.3-3$ is caused by the deprotonation and the decrease in the range 
below 1.3 by the addition of a proton to the zwitterion. Instead of a large plateau between these two processes only a shoulder in the curve is observed in the $\mathrm{pH}$ range $1-1.4$. Hence the $\mathrm{pK}_{\mathrm{a}}$ values of the carboxylate and the phosphate groups do not strongly differ from each other. Hence this confirms the spectroscopic results. It must be mentioned, however, that with respect to the proton transfer in the intramoleculear hydrogen bond in the zwitterion one can not distinguish strongly between a $\mathrm{pK}_{\mathrm{a}}$ of the carboxylate and of the phosphate groups.

In the case of the zwitterion the large polarisability of these hydrogen bonds leads to a coupling of numerous vibrations of the SPM molecule with the proton transitions in the hydrogen bonds which thus merge with the continuum. For instance, in the range $1500-1300 \mathrm{~cm}^{-1}$ most bands of the zwitterion which were observed with the $\mathrm{Na}^{+}$salt $(100 \%$ deprotonation) have disappeared. Apart from $\nu_{\mathrm{s}}$ of the $-\mathrm{CO}_{2}$ group at $1414 \mathrm{~cm}^{-1}$, only the asymmetrical bending vibration of the $-\mathrm{CH}_{2}$-group at $1468 \mathrm{~cm}^{-1}$ remains unchanged in the case of the zwitterion. This is understandable since the transition moment of this vibration is perpendicular to that of the $\mathrm{OH}$ stretching vibrations in the hydrogen bond, and thus does not couple with these vibration. In contrast to these observations, however, in the case of the zwitterion after $\mathrm{H} \rightarrow \mathrm{D}$ exchange all bands are found in the range $1500-1300 \mathrm{~cm}^{-1}$, as with the $\mathrm{Na}^{+}$salt. This is clear in view of the fact that asymmetrical $\mathrm{D}^{+}$hydrogen bonds with a double minimum potential well are not as strongly polarisable ${ }^{16}$. The transitions in these bonds therefore do not couple as strongly with the other vibrations of the molecule.

The absorbance of the continuous spectrum is plotted in Fig. 4 as a function of the degree of

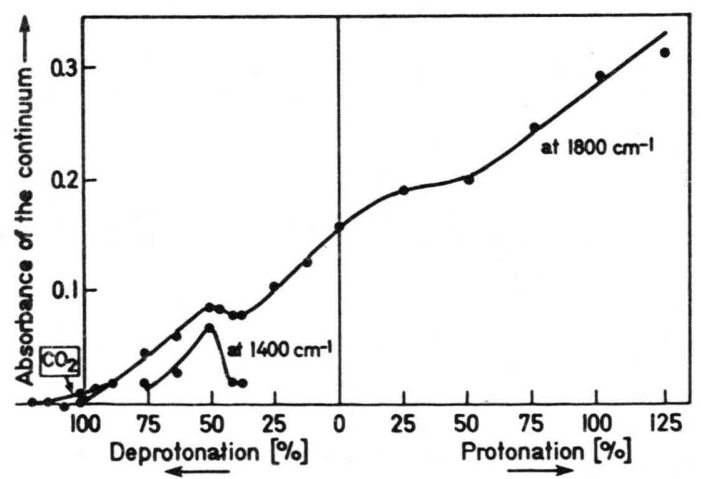

Fig. 4. Continuum of the SPM solution plotted over \% protonation or $\%$ deprotonation relative to the dissolved substance.

deprotonation and of protonation. In the range $0-40 \%$ deprotonation the absorbance of the conti- nuum decreases linearly with the deprotonation, that is, as the number of intramolecular hydrogen bonds decreases. The disappearance of these bonds can be traced by considering the bands, too. The $\mathrm{C}=\mathrm{O}$ and $\mathrm{P}=\mathrm{O}$ stretching vibrations at 1732 or $1252 \mathrm{~cm}^{-1}$, respectively, become less and less intense. In the spectrum of the $63 \%$ deprotonated sample, only $\nu \mathrm{C}=\mathrm{O}$ can still be recognised as a weak shoulder.

At about $50 \%$ deprotonation the absorbance increases slightly as the deprotonation decreases. Accordingly one now observes the formation of hydrogen bonds which make a greater contribution to the continuum than the asymmetrical ones. It appears that these are symmetrical intramolecular $\mathrm{OH}^{+} \cdots \mathrm{O}$ hydrogen bonds. Such symmetrical, easily polarisable hydrogen bonds must, however, supply a continuous absorption in the spectral range 1600$1200 \mathrm{~cm}^{-1}$, too ${ }^{9}$. This is indeed the case. The lower curve in Fig. 4 illustrates the variation of the absorbance of the continuous spectrum at about 1400 $\mathrm{cm}^{-1}$. The absorbance increases in this range, decreasing again as deprotonation increases.

At $100 \%$ deprotonation $\left(\mathrm{Na}^{+}\right.$salt $)$all easily polarisable hydrogen bonds have disappeared. Thus as the deprotonation increases the absorbance of the continuum undergoes no further change (Fig. 4). Instead, changes in the band of the asymmetrical stretching vibration of the $-\mathrm{NH}_{3}$ groups at 1528 $\mathrm{cm}^{-1}$ are observed (Fig. 1), for now these groups are transformed by the $\mathrm{OH}^{-}$ions to $-\mathrm{NH}_{2}$ groups. Strongly polarisable symmetrical $-\mathrm{NH}_{2} \mathrm{H}^{+} \cdot \cdots \mathrm{NH}_{2}$ bonds could hence form in the solution. However, it is known ${ }^{14}$ that these hydrogen bonds cause no continuum in the aqueous medium.

When investigating the range near $100 \%$ deprotonation in relation to the zwitterion, if the $\mathrm{CO}_{2}$ in the atmosphere is not excluded during preparation of the solutions a slightly different behaviour is observed (Fig. 4). The absorption of the continuum decreases more slowly, and instead the decrease extends over the range of deprotonation degrees above $100 \%$. This is due to the $\mathrm{HCO}_{3}$-ions present, which are formed by the $\mathrm{CO}_{2}$ and buffer the solution.

At about $700 \mathrm{~cm}^{-1}$ the torsional vibration of the water molecules is observed as an extremely broad and intense band. This intensity decreases as deprotonation of the SPM molecules increases, i.e. as the intramolecular hydrogen bond disappears on deprotonation. The molecules now disturb the water structure to a greater degree. This may explain the 
decreases observed in the intensity of the torsional vibration of the water molecules.

Can such hydrogen bonds be present under physiological conditions? One of the $\mathrm{pK}_{\mathrm{a}}$ values of the phospholipid phosphatidyl serine is observed at $4.4^{3}$. Hence it could be expected that the phosphate and carboxylic groups occur as anions near physiological $\mathrm{pH}$ values, i.e., that under these conditions the intramolecular hydrogen bonds cannot be observed. The $\mathrm{pK}_{\mathrm{a}}$ values, however, depend on the medium. Since the conditions under which the $\mathrm{pK}_{\mathrm{a}}$ values are measured differ from those in the membrane medium, it is, however, possible that such hydrogen bonds can be present in the biological membranes.

Let us now consider the protonation of the SPM. Fig. 1 shows that the $\mathrm{P}=\mathrm{O}$ stretching vibration at $1250 \mathrm{~cm}^{-1}$ becomes more intense as protonation increases, while the asymmetrical stretching vibration of the $>\mathrm{PO}_{2}$ groups at $1218 \mathrm{~cm}^{-1}$ becomes less intense. The asymmetrical stretching vibration of the $-\mathrm{CO}_{2}$ group disappears completely on protonation, which can be seen in the spectrum of the $\mathrm{D}_{2} \mathrm{O}$ solution. As expected, due to addition of the protons to the carboxylic or phosphate groups bonds with double or single bond character form. If the excess proton is added to the caroboxylic group, the proton is displaced in the intramolecular bond to the phosphate group. Hence in both groups one bond occurs with single and the other with double bond character. This takes place analogously when the excess proton is added to the phosphate group.

The continuum rises less rapidly in the range $0-50 \%$ as protonation increases. The $\mathrm{POH}^{+}$and $\mathrm{COH}^{+}$groups which form are strong hydrogen bond donors. Asymmetrical $\mathrm{POH}^{+} \cdots \mathrm{Cl}^{-}$or $\mathrm{COH}^{+} \cdots \mathrm{Cl}^{-}$ bonds form which evidently cause no continuum. At protonation degrees over $50 \%$ the intensity of the continuum again increases. Fig. 1 shows that this increase of the absorbance occurs in the range $1600-1000 \mathrm{~cm}^{-1}$, too. Accordingly, symmetrical, strongly polarisable hydrogen bonds form. This is understandable, for the solution now becomes acidic. $\mathrm{H}_{5} \mathrm{O}_{2}{ }^{+}$groupings form, whose extremely easily polarisable hydrogen bond causes the absorbance of the continuum to increase ${ }^{10,11}$. Similar curves have been investigated and discussed in detail for aqueous $\mathrm{N}$ base solutions ${ }^{14}$.

The extremely broad intense bands of the torsional vibration of the $\mathrm{H}_{2} \mathrm{O}$ molecules decrease in the range $0-127 \%$ protonation, as protonation increases. The same applies to the band at $2130 \mathrm{~cm}^{-1}$. This band is presumably a combination vibration of the scissor vibration and the torsional vibration ${ }^{17}$. This finding is understandable, since the water structure becomes disturbed as protonation increases. Furthermore, it is known ${ }^{9}$ that the transition moment of these vibrations couples with the transitions in the easily polarisable bonds. Hence this band merges with the continuum 9,14 .

The differences between the solutions of the different salts and the changes in the spectra resulting due to addition of counterion pair are slight. Thus only a few examples have been selected and are presented in Fig. 5.

Fig. 5 shows that the polarisable hydrogen bond in the zwitterion hardly changes when counterion pairs such as $\mathrm{KCl}$ or $\mathrm{CaCl}_{2}$ are present. This is illustrated particularly well by the spectra of the $\mathrm{D}_{2} \mathrm{O}$ solutions in Fig. $5 \mathrm{~b}$. The band of the $\mathrm{C}=\mathrm{O}$ stretching vibration is intense in the presence of $\mathrm{CaCl}_{2}$ as well as of $\mathrm{KCl}$. The decreases in $\mathrm{pH}$, particularly as observed in the presence of $\mathrm{Ca}^{2+}$ ions with phospholipids $^{2,5}$, are accordingly too weak as to be noticed in the IR spectra.

Fig. $5 \mathrm{c}$ and $\mathrm{d}$ show spectra of different salts in $\mathrm{H}_{2} \mathrm{O}$ and $\mathrm{D}_{2} \mathrm{O}$ solutions. It can be seen from Fig. $5 \mathrm{~d}$ that the band at $1738 \mathrm{~cm}^{-1}$, i.e., $\nu \mathrm{C}=\mathrm{O}$ in the $\mathrm{Ca}^{2+}$ sample', has not disappeared completely. When producing this salt in the ion exchanger the protons were not completely removed by the $\mathrm{Ca}^{2+}$ ions, in contrast to observations made with all other cations, particularly with the $\mathrm{Mg}^{2+}$ ions.

Further, the spectra of the $\mathrm{K}^{+}$and $\mathrm{Ca}^{2+}$ salts scarcely differ from each other. With some bands, however, differences are observed in comparing these salts with the zwitterion. Fig. $5 \mathrm{c}$ shows that the band of the asymmetric $-\mathrm{NH}_{3}$ bending vibration at $1528 \mathrm{~cm}^{-1}$ is shifted somewhat toward larger wave numbers, whereas the band of the $\mathrm{CN}$ stretching vibration at $1328 \mathrm{~cm}^{-1}$ migrates slightly toward smaller wave numbers on transition to the zwitterion Accordingly, with the zwitterion the $\mathrm{CN}$ bond is somewhat looser. Furthermore, the hydrogen bonds formed by the $-\mathrm{NH}_{3}$ group are stronger, because the $\mathrm{NH}$ bonds in this group are weaker. Both observations can be understood by considering that the presence of the proton at the carboxylic group leads to an electron shift in the molecule, and especially to shielding of the negative charge of the carboxylic group. 

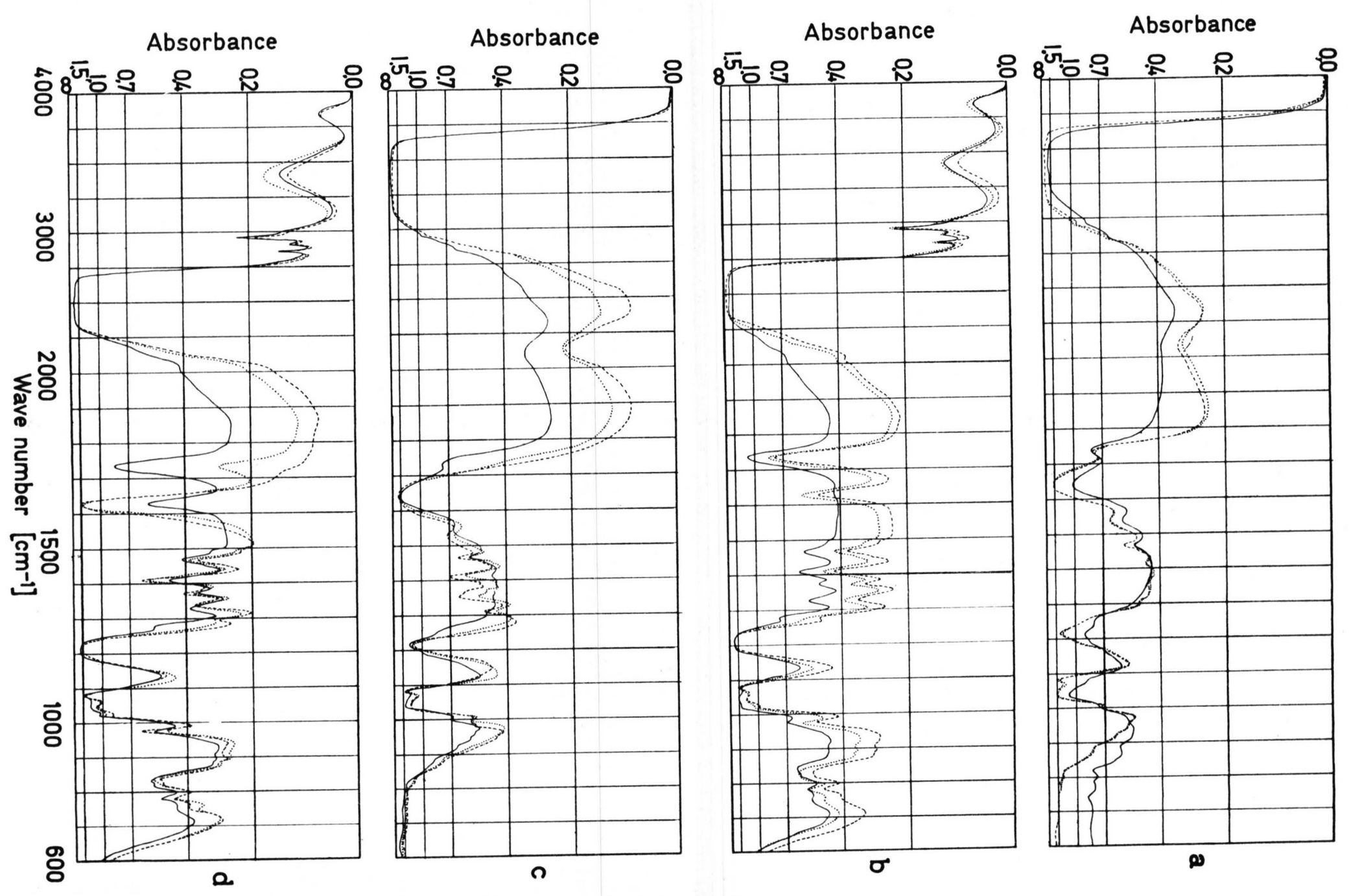

Fig. 5. IR Spectra of aqueous solutions of SPM; a. zwitterion, — - with $\mathrm{HCl}$ counterion pair, -..- with $\mathrm{KCl}$ counterion pair, $\cdots$ with $\mathrm{CaCl}{ }_{2}$ counterion pair; $b$. the same as with a. but $\mathrm{D}_{2} \mathrm{O}$ solutions; $c$. - zwitterion, - -- $\mathrm{K}^{+}$salt, $\cdots \mathrm{Ca}^{2+}$ salt; d. the same as with c. but $\mathrm{D}_{2} \mathrm{O}$ solution. 
The $\mathrm{NH}$ groups of the $-\mathrm{NH}_{3}$ group accordingly become stronger hydrogen bond donors when the carboxylic group is partially shielded. This is consistent with the finding of ABRAMSON et al. ${ }^{2}$, that is, that the suspensions of the $\mathrm{Na}^{+}$and $\mathrm{K}^{+}$salts of the corresponding phospholipids become somewhat acidic on the addition of neutral salt. These authors give the explanation that on the addition of neutral salt some protons become detached from the $-\mathrm{NH}_{3}$ groups. The addition of neutral salt leads to better shielding of the carboxylic group and thus - connected with the increase of the hydrogen bond donor property of the $-\mathrm{NH}_{3}$ group - to slight preference of proton detachment.

\section{II.2. Ethanolamine phosphoric acid methylester}

Fig. 6 shows spectra of aqueous EPM solutions as a function of the protonation. As protonation increases a continuous absorption forms, which begins at $3000 \mathrm{~cm}^{-1}$ and extends toward small wave numbers over the entire range investigated. Such a continuum indicates the presence of symmetrical, extremely easily polarisable hydrogen bonds.

The $\mathrm{pK}_{\mathrm{a}}$ value of the EPM molecules is 1.15 , like that of the phosphatidyl ethanolamine $(1.1)^{6}$ and is thus not much larger than the $\mathrm{pK}_{\mathbf{a}}$ (proton gain) of the water molecules ${ }^{18}$. It is known from investigations of the aqueous $\mathrm{N}$ base systems ${ }^{14}$ that under these conditions symmetrical, easily polarisable hydrogen bonds first form between the dissolved molecules. Thus at low degrees of protonation easily polarisable $\mathrm{POH}^{+} \ldots$ OP hydrogen bonds occur. A symmetrical hydrogen bond between the phosphate groups of the di- $p$-chlorophenyl hydrogen phosphate has been investigated by HADžI and NovAK in the solid state ${ }^{19}$. At higher degrees of protonation the rise of the continuum is caused preferably by the formation of $\mathrm{H}_{5} \mathrm{O}_{2}$.

Furthermore, aqueous solutions of the EPM zwitterion were investigated in the presence of counterion pairs such as $\mathrm{NaCl}, \mathrm{KCl}, \mathrm{CaCl}_{2}$ and $\mathrm{MgCl}_{2}$. These counterion pairs cause very little
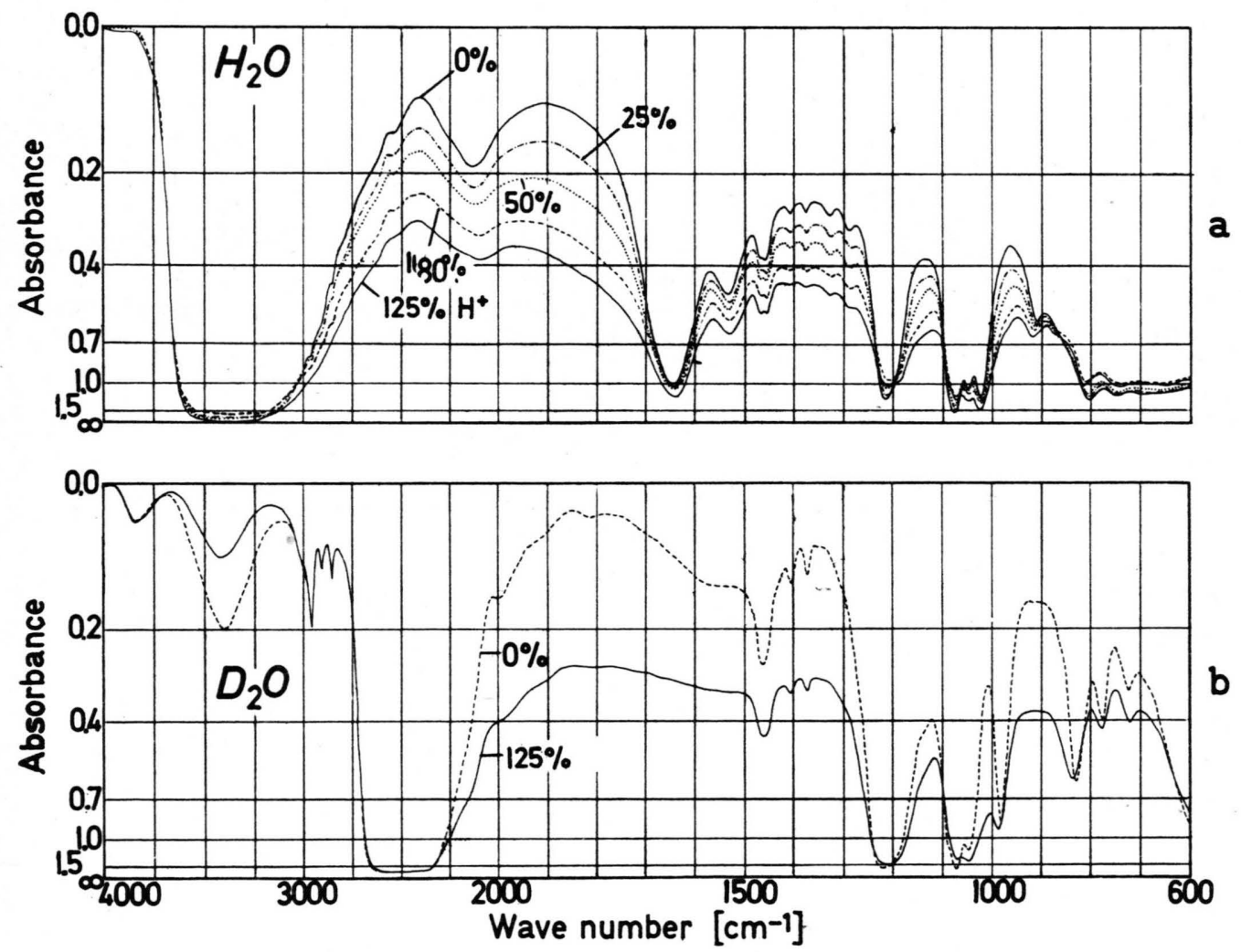

Fig. 6. IR Spectra of aqueous etanolamine phosphoric acid P-methylester solutions as a function of $\%$ protonation with $\mathrm{HCl}$ relative to the dissolved substance; $\mathrm{a} . \mathrm{H}_{2} \mathrm{O}$ solution; $\mathrm{b} . \mathrm{D}_{2} \mathrm{O}$ solution. 
change in the spectrum. It can only be observed that the asymmetric bending vibration of the $-\mathrm{NH}_{3}$ group shifts in the presence of the counterions by about $4 \mathrm{~cm}^{-1}$ toward greater wave numbers. This shift is presumably caused by the formation of $\mathrm{NH}^{+} \ldots \mathrm{Cl}^{-}$bonds. This demonstrates that the phosphatidyl ethanolamine can not be responsible for the cation selectivities in biological membranes.

\section{Experimental Procedure}

The serine phosphoric acid P-methylester and the ethanolamin phosphoric acid P-methylester were synthesised according to a procedure described in? These substances can now be supplied by the firm of Roth, Karlsruhe, W. Germany. The samples were deprotonated by addition of various amounts $\mathrm{NaOH}$ to the solution of the zwitterion and protonated by addition of various amounts $\mathrm{HCl}$. The $100 \%$ deprotonated sample is the $\mathrm{Na}^{+}$salt. The different salts were produced from the zwitterion via ion exchangers. We obtained the samples with additional counterion pairs by addition of the same amounts of the corresponding salts. The concentration of the model molecules in the solutions was $480 \mathrm{mg} / \mathrm{ml}$ during the investigations.

The samples were placed in a cell with $\mathrm{Ge}$ windows, as described in ${ }^{13}$, for plotting the IR spectra. The sample temperature was $25^{\circ} \mathrm{C}$. The spectra were plotted with the IR spectrophotometer model 325 of the Perkin-Elmer Bodenseewerk Company, Ueberlingen, W. Germany (slit programme 6.5, gain 2.5, response 2).
We thank the Deutsche Forschungsgemeinschaft and the Fond der Chemischen Industrie for providing the facilities for this work and the Deutsche Akademische Austauschdienst for a grant for one of us (G. P.).

1 G. Foelsch and R. Oesterberg, J. biol. Chemistry 234, 2298 [1959].

2 M. B. Abramson, R. Katzman, and H.P. Gregor, J. biol. Chemistry 239, 70 [1964].

3 G.H. De Haas, H. van Zutphen, P.P. M. Bonsen, and L.L.M. van DeEnen, Recueil 83, 99 [1964].

4 E. Rojas and J.M. Tobias, Biochim. biophysica Acta [Amsterdam] 94, 394 [1965].

5 M.P. Blaustein, Biochim. biophysica Acta [Amsterdam] 135, 653 [1967].

6 E. Junger, Histochemie 13, 296 [1968].

7 G. Papakostidis, Thesis Munich University 1972

8 E. G. Weidemann and G. Zundel, Z. Naturforsch. 25 a, 627 [1970].

9 R. Janoschek, E. G. Weidemann, H. Pfeifer, and G. ZundeL, J. Amer. chem. Soc. 94, 2387 [1972].

10 G. ZUNDEL, Hydration and Intermolecular Interaction, Academic Press, New York 1969, and Mir, Moskwa 1972.

11 G. Zundel and H. Metzger, Z. physik. Chem. [Frankfurt] 58, 225 [1968].

12 G. Zundel, Allgem. Prakt. Chem. [Wien] 21, 329 [1970].

13 G. Zundel and J. Muehlinghaus, Z. Naturforsch. 26b, 546 [1971].

14 W. Sessler and G. Zundel. Z. physik. Chem. 79, 180 [1972].

15 W. SEsSLER and G. Zundel, Chem. Phys. Letters 14,356 [1972].

16 R. JANOSCHEK, private communication.

17 G.E. Walrafen, J. chem. Physics 36, 1035 [1962].

18 E.M. Arnetr, Prog. Phys. Org. Chem. 1, 283 [1963].

19 D. HADži and A. NovaK, Proc. Chem. Soc. 1960, 241. 